\title{
Sensitivity enhancement of plasmonic grating in near field
}

\author{
L.L. Frumin, D.A. Shapiro" \\ Institute of Automation and Electrometry, Siberian Branch, Russian Academy of Sciences \\ *E-mail: shapiro@iae.nsk.su
}

DOI: $10.31868 /$ RFL2020.26-27

As predicted and tested experimentally by Liedberg [1], under proper conditions, the reflectivity of a thin metal film is extremely sensitive to optical variations in the medium on one side of it. Among devices developed recently, the sensors based on the surface plasmons are distinguished (see [2,3]). A plane wave falls to the boundary with an analyte from the glass. Its incident angle is close to the total internal reflection. A metallic layer or a plasmon-supporting sub-wavelength grating at the interface makes the reflection coefficient's dependence on the incidence angle (or the wavelength) sensitive to the refractive index of the analyte.

The purpose of the present paper is to study a way to increase sensitivity. We propose to measure the local field in points where the field enhancement factor $F_{1}=\left|E_{x} / E_{0}\right|^{2}$ of a grating being maximal. Here $E_{x}$ is the tangential component of the electric field, $E_{0}$ is the amplitude of an incident wave. For this purpose, we consider the subwavelength periodic sequence of metallic cylinders placed above the dielectric halfspace $\varepsilon_{1}$, shown in Fig.1. An analyte with refractive index $n_{a}$ fills half-space $\varepsilon_{2}=n_{a}^{2}$.

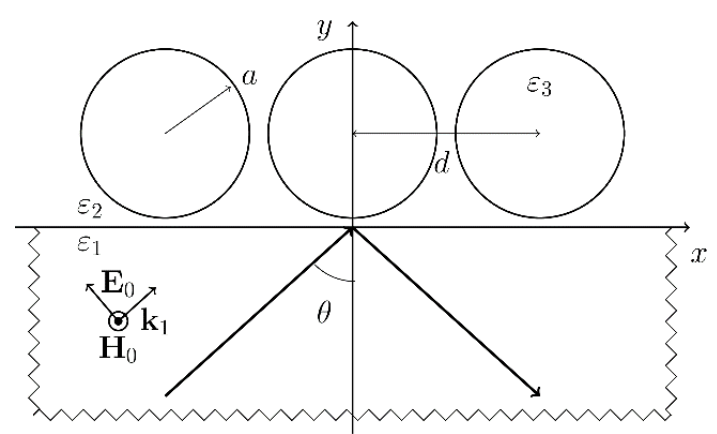

Fig. 1. The sketch of medium 2, above the dielectric subspace 1 . The circular cylinders of radius $a$ form the grating with period $d$. Inset illustrates the polarization state of $p$-wave.

The approximate analytical formulas could be derived using the coupled-mode approach [4]. Here we calculate the field numerically by the boundary element method [5]. One can see some typical results of numerical modeling in Fig. 2. Figure 2(a) demonstrates the shift of resonance in angular dependence for a gold grating for two close refractive indices. We see a significant shift after changing $n_{a}$ by $1 \%$ only. The location of peak at $\varepsilon_{1}=2.25$ corresponds to angles $\theta_{0}=0.7297\left(n_{a}=1\right)$ or $\theta_{0}=$ $0.7342\left(n_{a}=1.01\right)$.

Figure 2(b) occurs more indicative. It displays the shift of angular resonance for a silver grating while the index changes by $0.1 \%$. We take the refractive index from the Polyanskiy database [6]. In both figures, we see the sharp cusp-like resonances near the angle of total reflection. The cusp is a consequence of the Fresnel field angular dependence [7] shown in Fig.3. Its small-angle tail is unusually steep; for an unperturbed dielectric-analyte interface, it has the infinite derivative from left side. This dependence leads to high sensitivity to the analyte index. 


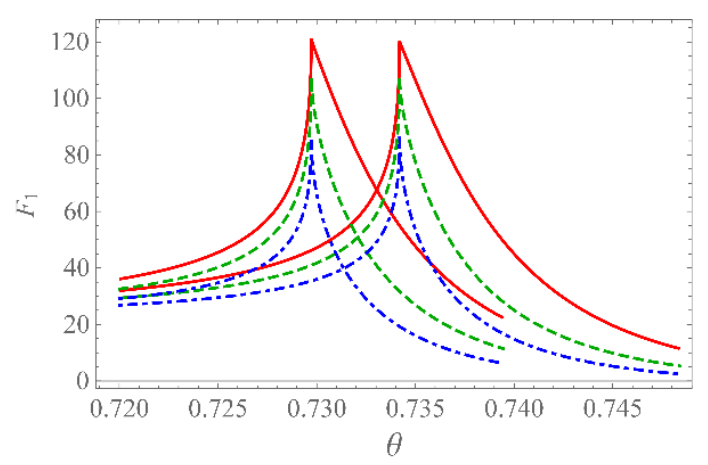

(a)

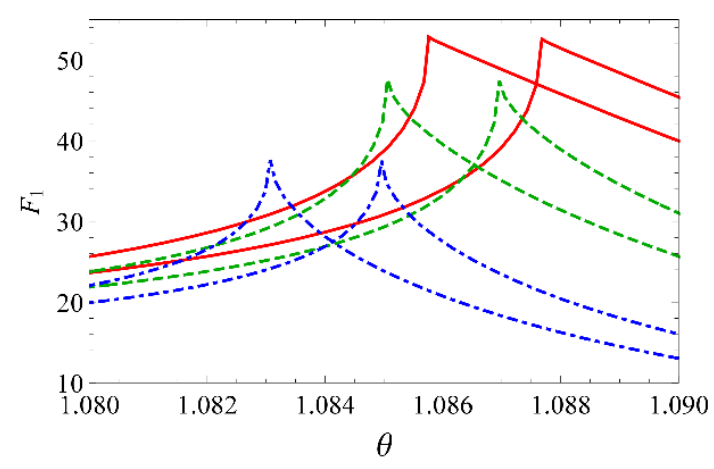

(b)

Fig. 2. Angular dependence of the local field in the gap for a gold grating in gas (a): $\lambda=$ 0.7749 (solid line), 0.8266 (dashed), $0.8856 \mu \mathrm{m}$ (dot-dashed) $n_{a}=1.00$ at the left, $n_{a}=$ 1.01 at the right and silver lattice; for silver grating in water (b): $\lambda=0.9537$ (solid line), 1.033 (dashed), $1.127 \mu \mathrm{m}$ (dot-dashed) $n_{a}=1.3270$. 1.3265. 1.3251, respectively, for the left plots, $n_{a}$ is greater by $0.001 n_{a}$ for the right curves.

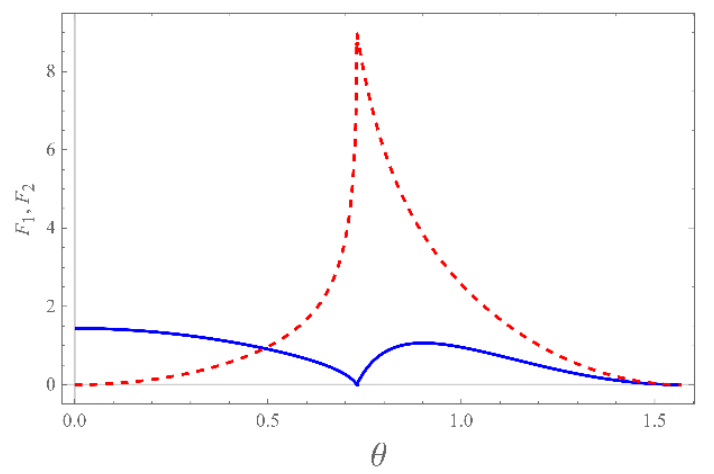

Fig. 3. The Fresnel angular dependence near the plane normalized by the incident field: $F_{1}=$ $\left|E_{x} / E_{0}\right|^{2}$ (solid), $F_{2}=\left|E_{y} / E_{0}\right|^{2}$ (dashed line).

We suggest applying a grating of parallel metallic cylinders on the glass substrate to increase the sensitivity of optical refractive index measurements. It is helpful to measure the field between neighbor cylinders. This signal is strong owing to the gap plasmon amplification. Moreover, its angular dependence has a very sharp cusp, which is a consequence of the total internal reflection. Both the factors are essential for further potential exploiting the subwavelength metallic grating in chemo- and bio-sensing techniques.

The authors are grateful to V.P. Drachev and S. V. Perminov for helpful discussions. The work is supported by the Russian Foundation of Basic Research, grant \# 20-02-00211.

\section{References}

[1] B. Liedberg, C. Nylander, I. Lunström, Sensors and actuators. 4, 299-304 (1983)

[2] Y. Xu et al., Adv. Opt. Mater. 7, 1801433 (2019)

[3] T. Allsop, R. Neal, Sensors 19, 4874 (2019).

[4] E. Efremova, S. Perminov, S. S. Vergeles, arXiv: 1905.04784

[5] L. L. Frumin et al., J. Opt. 15, 085002 (2013)

[6] M. N. Polyanskiy, "Refractive index database," https://refractiveindex.info.

[7] L. D. Landau and E. M. Lifshitz, Electrodynamics of continuous media (Pergamon, Oxford, New York, 1984). 